\title{
Call Admission Control for 3G CDMA Networks with Differentiated QoS
}

\author{
Qian Huang ${ }^{1}$, Hui Min Chen ${ }^{1}$, King Tim $\mathrm{Ko}^{2}$, Sammy Chan ${ }^{2}$, and \\ King Sun Chan $^{3}$
}

1 School of Communication and Information Engineering, Shanghai University

No. 149, Yan Chang Road, 200072 Shanghai, China

hqian@online.sh.cn

2 Department of Electronic Engineering, City University of Hong Kong

Tat Chee Avenue, Kowloon, Hong Kong

\{eektko, eeschan\}@cityu.edu.hk

3 Department of Electrical and Electronic Engineering, The University of Hong Kong

Pokfulam Road, Hong Kong

kschan@eee.hku.hk

\begin{abstract}
A call admission control (CAC) scheme for 3G wireless networks based on the estimated interference and differentiated quality of service (QoS) is proposed in this paper. In this CAC scheme, the interferences introduced by the to-be-admitted new call and its impact to the QoS of the existing connections in the adjacent cells are considered in call admission. The purpose is to obtain QoS balance amongst neighboring cells within a cluster. Comparison of our proposed scheme with the current scheme without considering the effects of the to-be-admitted calls indicates that the proposed scheme performs better in outage and blocking probabilities.
\end{abstract}

\section{Introduction}

The third-generation $(3 \mathrm{G})$ network will support broadband applications like streaming video, web browsing and network games via wireless IP [1]. As the radio link is bandwidth limited and error prone, wireless IP applications face some major challenges in practice. QoS is a crucial issue in delivering the services. To guarantee the QoS for different services over the radio link, admission control schemes that can handle differentiated QoS and efficiently utilize the scarce bandwidth are important.

Some admission control schemes for the CDMA based networks have been studied in [2]-[5]. The CAC schemes presented in [2] and [3] were similar: a new call accessed the network with its target signal-to-interference ratio (SIR) requirement; both of the proposed schemes accepted the new call in terms of the estimated residual capacity over the radio link. However, since these two schemes handled the case of single type of voice traffic, the homogeneous QoS requirement on SIR was only considered in CAC. 
More recent studies on CAC schemes suitable for a system with different services are made in [4] and [5]. These CAC schemes are based on the total tolerable interference in a cell. The CAC scheme presented in [4] focused on the single cell system model, in which the interference from the adjacent cells was neglected. Shen and Ji 5] considered a cell cluster system in their proposed CAC scheme. When a new call arrived, the interference levels from other cells and the path loss experienced by the new call were estimated; if the total equivalent bandwidth taken up by all users in the cell, including the to-be-admitted new call, plus the estimated inter-cell interference exceeded the maximal channel capacity, the new call will be blocked, otherwise, it will be admitted.

However, the scheme in [5] did not consider the QoS degradation and unbalance in the adjacent cells caused by the admitted calls in the serving cell. Particularly, if a new call with high bit rate is admitted at the edge of its serving cell and, its adjacent cells are heavily loaded at the same time, the on-going connections in these adjacent cells may experience QoS degradation and communication outage because of the increase in the interference level. Generally, the on-going connections outage is more unacceptable than the blocking of a new call. In order to reduce the outage probability of the on-going connection and obtain balanced QoS within the neighboring cells, we propose here a new CAC scheme in which the interference introduced by the to-be-admitted call is considered during call admission in both the serving and adjacent cells. A new call will be admitted, if the total estimated resultant interferences in both serving and adjacent cells are under a predefined threshold, so that the guaranteed QoS of the existing connections within the cell cluster will not be violated after the new call is admitted.

In Section 2, the model for our proposed call admission scheme is presented. Section 3 derives the outage and blocking probabilities of the proposed admission control scheme for different service types. Section 4 gives the numerical results and comparisons in performances with an existing admission scheme. The final section gives the concluding remarks.

\section{$2 \quad$ System Model}

In this paper, we consider the scenario of differentiated QoS provisioned over a cluster of cells system, in which the cell of interest is surrounded by $H$ adjacent cells $(H=6)$. Services are classified in terms of the requirements of BER and transmission rate. Two typical IP traffics are assumed in our proposal. One is the $8 \mathrm{kbps}$ packet voice, the other is the WWW traffic with average rate equal to $144 \mathrm{kbps}$. For the packet voice traffic, the ON-OFF model is used. For the WWW traffic, the traffic model proposed in [9] is adopted. The size of a web page has a log-normal distribution, and the thinking interval has an exponential distribution. The call arrival is assumed to follow Poisson distribution for both voice and data services.

The multi-code mode is adopted in our model to support different bit rate services. After a call is admitted, more than one code is allocated for the ag- 
gregated transmission rate. Each code is equivalent to a sub-channel, the high speed data service is transported simultaneously over several sub-channels and the equal spreading gain is achieved on each sub-channel. Let $m_{d}$ be the number of sub-channels for a call, let $R_{b}$ be the basic bandwidth of the sub-channel, thus the aggregate transmission rate equals to $R_{b} \cdot m_{d}$.

It is assumed that the background noise is additive white Gaussian noise (AWGN). For the total interference levels from the voice and data users in the neighboring cells, they are also approximated by the Gaussian distribution as in 6]. With only a few high rate users, the interference distribution might not be Gaussian, however we are making the above assumption for the common distribution of high and low rate users within cells. Let $I_{v}^{i}, I_{d}^{i}, I_{v}^{h}$ and $I_{d}^{h}$ respectively be the interference introduced to the cell $i$ and $h$ from the voice and data users in their adjacent cells, their mean and variance can be obtained according to the path loss model used in [6]:

$$
\text { Path loss }=10^{(\xi / 10)} r^{-4},
$$

where $r$ is the distance from mobile station to the target BS, $\xi$ is approximated as a Gaussian random variable with zero mean and $8 \mathrm{~dB}$ variance. For a mobile station (MS) $j$, assuming its serving BS is $i$, and adjacent BS is $h$. Let the received power of MS $j$ at BS $i$ be represented as $S_{v}$ for the voice service and $S_{d}$ for the data service. From Equation (1), we can obtain that the interference power level received at BS $h$ from MS $j$ is $S_{v} \frac{l_{j}(h)}{l_{j}(i)}$ and $S_{d} \frac{l_{j}(h)}{l_{j}(i)}$ for the voice and data call respectively, where $l_{j}(h)$ represents the path loss from the BS $h$ to MS $j, l_{j}(h)=10^{\left(\xi_{h} / 10\right)} r_{j h}^{-4}$; and $l_{j}(i)$ represents the path loss from MS $j$ to its serving $\mathrm{BS} i, l_{j}(i)=10^{\left(\xi_{i} / 10\right)} r_{j i}^{-4}$.

We also assume that perfect power control is implemented on the up-link, and equal power can be received at the BS from each user of the same traffic class. Let $S I R_{t h}^{v}$ be the target SIR for the voice service, and $S I R_{t h}^{d}$ for the data service, the target SIR values are corresponding to the BER requirements. Let $R_{v}$ be the peak bit rate for the voice, and $R_{d}$ be the average bit rate for the data, $R_{d}=m_{d} R_{b}$. The product of the target SIR and the transmission rate is defined as the equivalent bandwidth requested by the differentiated QoS, that is $B_{v}=S I R_{t h}^{v} R_{v}$ for the voice service, and $B_{d}=S I R_{t h}^{d} R_{d}$.

Given the radio link bandwidth $(w)$, the maximum total acceptable interference density $\left(I_{0}\right)$ within a CDMA cell, including the inter-cell interference, proves 10 times as high as that of the background noise $\left(N_{0}\right)$, that is $I_{0}=10 N_{0}$ [7]. Let $a_{j}^{v}$ represent the voice user $j$ 's activity factor, its mean is denoted as $\alpha_{v}$, $\alpha_{v}$ is assumed to be $3 / 8$ [6]; let $a_{j}^{d}$ be the activity factor of user $j$ with WWW traffic, and $\alpha_{d}$ be the mean. Based on the above predefined threshold, when a new call with transmission rate $R_{\text {new }}$ and target SIR $S I R_{t h}^{\text {new }}$ arrives at its serving BS $i$, the CAC scheme will check whether the interferences measured at BS $i$ and the adjacent $\mathrm{BS} h(h=1,2, \ldots, H)$ violate the following two admission conditions: For BS $i$, the condition is

$$
B_{\text {new }}+\sum_{j=1}^{N_{v}^{i}} a_{j}^{v} B_{v}+\sum_{j=1}^{N_{d}^{i}} a_{j}^{d} B_{d}+\frac{I_{v}}{I_{0}}+\frac{I_{d}}{I_{0}} \leq 0.9 w
$$


where $B_{\text {new }}=S I R_{t h}^{\text {new }} R_{\text {new }}$ is the equivalent bandwidth requested by the new call; $N_{v}^{i}$ and $N_{d}^{i}$ respectively represent the number of existing voice and data calls in the serving cell $i ; a_{j}^{v}$ and $a_{j}^{d}$ represent the activity factor of the $j^{\text {th }}$ voice call and data call in cell $i ; I_{v}$ and $I_{d}$ denote the interference powers from the voice and data calls in the adjacent cells; the levels of $I_{v}$ and $I_{d}$ are approximated by a Gaussian distribution.

For BS $h$, the interference power introduced by the signal power of the new call received at the BS $i\left(S_{\text {new }}^{i}\right)$ to the BS $h$ can be obtained by the ratio of the path-loss from mobile station (MS) $j$ to BS $h\left(l_{j}(h)\right)$ to that from MS $j$ to BS $i$ $\left(l_{j}(i)\right)$, then the admission condition is

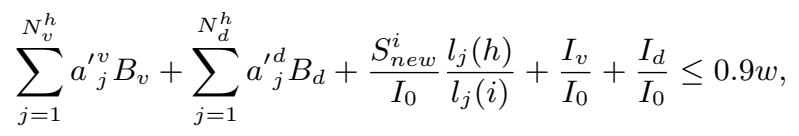

where $N_{v}^{h}$ and $N_{d}^{h}$ respectively indicate the number of existing voice and data calls in the adjacent cell $h ; a_{j}^{\prime v}$ and ${a^{\prime}}_{j}^{d}$ represent the activity factor of the $j^{\text {th }}$ voice call and data call in cell $h$. When both Equation (2) and (3) are satisfied, the new call is accepted; otherwise, it is blocked.

\section{Derivation of Outage and Blocking Probabilities}

In the CDMA-based network, the probability of the received SIR lower than the target SIR is defined as the outage probability, which indicates the QoS performance supported by the network, while the blocking probability is related to the network capacity. Here, we use these two parameters as the criteria in assessing our admission control scheme.

The CDMA reverse link model for calculating the SIR by received voice users is given by Gilhousen et al. [6]. Based on this single traffic model, Ayyagari and Ephremides developed a model for integrated voice and data services in [8]. In our analysis, the reverse link model proposed in 8 is adopted. The difference is that the interference caused by the to-be-admitted call to its adjacent cells is included in our model.

The call arrivals for both voice and data services follow Poisson processes with mean arrival rate $\lambda_{v}$ and $\lambda_{d}$ respectively; let $x$ and $y$ denote the number of arrived calls during the average service time $T_{s}$ for the voice and data respectively. Let $S I R_{i}^{v}$ be the received SIR at the serving BS $i$ from a voice call, and $S I R_{i}^{d}$ for the data call, the received SIR for an on-going voice and data call in the serving cell $i$ can be derived as follows:

$$
\operatorname{SIR}_{i}^{v}=\frac{w / R_{b}}{\sum_{j=1}^{N_{v}^{i}-1} a_{j}^{v}+\sum_{j=1}^{N_{d}^{i}} a_{j}^{d} m_{d} \frac{S_{d}}{S_{v}}+x+y m_{d} \frac{S_{d}}{S_{v}}+\frac{I_{v}^{i}+I_{d}^{i}+N_{0} w}{S_{v}}}
$$




$$
S I R_{i}^{d}=\frac{w / R_{b}}{\sum_{j=1}^{N_{v}^{i}} a_{j}^{v} \frac{S_{v}}{S_{d}}+\sum_{j=1}^{N_{d}^{i}-1} a_{j}^{d} m_{d}+\left(m_{d}-1\right)+x \frac{S_{v}}{S_{d}}+y m_{d}+\frac{I_{v}^{i}+I_{d}^{i}+N_{0} w}{S_{d}}}
$$

Considering the interference from the to-be-admitted call in serving cell $i$, the received SIR at $\mathrm{BS} h$, can be expressed as follows:

$$
\begin{aligned}
S I R_{h}^{v} & =\frac{w / R_{b}}{\sum_{j=1}^{N_{v}^{h}-1} a_{j}^{\prime v}+\sum_{j=1}^{N_{d}^{h}} \frac{a_{j}^{\prime}{ }_{j} m_{d} S_{d}}{S_{v}}+\sum_{j=1}^{x} \frac{l_{j}(h)}{l_{j}(i)}+\sum_{j=1}^{y} \frac{l_{j}(h)}{l_{j}(i)} \frac{m_{d} S_{d}}{S_{v}}+\frac{I_{v}^{h}+I_{d}^{h}+N_{0} w}{S_{v}}} \\
S I R_{h}^{d}= & \frac{w / R_{b}}{\sum_{j=1}^{N_{v}^{h}} a_{j}^{\prime v} \frac{S_{v}}{S_{d}}+\sum_{j=1}^{N_{d}^{h}-1} a_{j}^{\prime}{ }_{j} m_{d}+\left(m_{d}-1\right)+\sum_{j=1}^{x} \frac{l_{j}(h) S_{v}}{l_{j}(i) S_{d}}+\sum_{j=1}^{y} \frac{m_{d} l_{j}(h)}{l_{j}(i)}+\frac{I_{v}^{h}+I_{d}^{h}+N_{0} w}{S_{d}}}
\end{aligned}
$$

In the above equations, the mean and the variance of $I_{v}^{i} / S_{v}$ and $I_{d}^{i} / S_{d}$ can be derived as in [6]:

$$
\begin{gathered}
E\left(I_{v}^{i} / S_{v}\right) \leq 0.247 N_{v}^{i} \cong \mu_{v} \\
E\left(I_{d}^{i} / S_{d}\right) \leq 0.247 N_{d}^{i} m_{d} \frac{\alpha_{d}}{\alpha_{v}} \cong \mu_{d} \\
\operatorname{var}\left(I_{v}^{i} / S_{v}\right) \leq 0.078 N_{v}^{i} \cong \sigma_{v}^{2} \\
\operatorname{var}\left(I_{d}^{i} / S_{d}\right) \leq 0.078 N_{d}^{i} m_{d}^{2} \frac{\alpha_{d}}{\alpha_{v}} \cong \sigma_{d}^{2}
\end{gathered}
$$

where $\alpha_{v}$ and $\alpha_{d}$ are the means of voice and data activity factor respectively.

From Equation (6) and (7), the interference levels received at the BS $h$ from the to-be-admitted $x$ and $y$ calls in the cell $i$, are equivalent to the increase of the user number of $x$ and $y$ in the cell $h$. Let $I_{v}^{\prime} / S=\sum_{j=1}^{x} \frac{l_{j}(h)}{l_{j}(i)}+\frac{I_{v}^{h}}{S}, I_{d}^{\prime} / S=$ $\sum_{j=1}^{y} \frac{l_{j}(h)}{l_{j}(i)} m_{d}+\frac{I_{d}^{h}}{S}$, then we have,

$$
\begin{gathered}
E\left(I_{v}^{\prime} / S_{v}\right) \leq 0.247\left(N_{v}^{h}+\lambda_{v} T_{s}\right) \cong \mu_{v}^{\prime} \\
E\left(I_{d}^{\prime} / S_{d}\right) \leq 0.247\left(N_{d}^{h}+\lambda_{d} T_{s}\right) m_{d} \frac{\alpha_{d}}{\alpha_{v}} \cong \mu_{d}^{\prime} \\
\operatorname{var}\left(I_{v}^{\prime} / S_{v}\right) \leq 0.078\left(N_{v}^{h}+\lambda_{v} T_{s}\right) \cong \sigma_{v}^{\prime 2} \\
\operatorname{var}\left(I_{d}^{\prime} / S_{d}\right) \leq 0.078\left(N_{d}^{h}+\lambda_{d} T_{s}\right) m_{d}^{2} \frac{\alpha_{d}}{\alpha_{v}} \cong \sigma_{d}^{\prime 2}
\end{gathered}
$$


From Equation (4) and (5), we can derive the outage probability for the differentiated QoS at the serving BS $i$ :

$$
\begin{aligned}
P_{\text {iout }}^{v}= & \operatorname{Pr}\left(S I R_{i}^{v} \leq S I R_{\text {th }}^{v}\right) \\
= & \operatorname{Pr}\left(\sum_{j=1}^{N_{v}^{i}-1} a_{j}^{v}+\sum_{j=1}^{N_{d}^{i}} a_{j}^{d} m_{d} \frac{S_{d}}{S_{v}}+x+y m_{d} \frac{S_{d}}{S_{v}}+\frac{I_{v}^{i}+I_{d}^{i}+N_{0}}{S_{v}} \geq \frac{w / R_{b}}{S I R_{\text {th }}^{v}}\right) \\
= & \operatorname{Pr}\left(x+y m_{d} \frac{S_{d}}{S_{v}}+\frac{I_{v}^{i}+I_{d}^{i}}{S_{v}} \geq \frac{w / R_{b}}{S I R_{t h}^{v}}-\frac{N_{0}}{S_{v}}-k_{v}-k_{d} m_{d} \frac{S_{d}}{S_{v}}\right) \\
& \cdot\left[\sum_{k_{d}=0}^{N_{d}^{i}} \sum_{k_{v}=0}^{N_{v}^{i}-1}\left(\begin{array}{c}
N_{d}^{i} \\
k_{d}
\end{array}\right)\left(\begin{array}{c}
N_{v}^{i}-1 \\
k_{v}
\end{array}\right) \alpha_{v}^{k_{v}}\left(1-\alpha_{v}\right)^{N_{v}^{i}-1-k_{v}} \alpha_{d}^{k_{d}}\left(1-\alpha_{d}\right)^{N_{d}^{i}-k_{d}}\right] \\
= & \sum_{k_{d}=0}^{N_{d}^{i}} \sum_{k_{v}=0}^{N_{v}^{i}-1}\left(\begin{array}{c}
N_{d}^{i} \\
k_{d}
\end{array}\right)\left(\begin{array}{c}
N_{v}^{i}-1 \\
k_{v}
\end{array}\right) \alpha_{v}^{k_{v}}\left(1-\alpha_{v}\right)^{N_{v}^{i}-1-k_{v}} \alpha_{d}^{k_{d}}\left(1-\alpha_{d}\right)^{N_{d}^{i}-k_{d}} \\
& \cdot Q\left(\frac{\mu_{1}}{\sqrt{\sigma_{1}^{2}+\sigma_{2}^{2}}}\right)
\end{aligned}
$$

where,

$$
\begin{aligned}
& \mu_{1}=\frac{w / R_{b}}{S I R_{t h}^{v}}-\frac{N_{0}}{S_{v}}-k_{v}-k_{d} m_{d}-\left(\mu_{v}+\mu_{d} \frac{S_{d}}{S_{v}}\right)-\lambda_{v} T_{s}-\lambda_{d} T_{s} m_{d} \frac{S_{d}}{S_{v}} \\
& \sigma_{1}^{2}=\lambda_{v} T_{s}+\sigma_{v}^{2} \\
& \sigma_{2}^{2}=\left(\lambda_{d} T_{s} m_{d}^{2}+\sigma_{d}^{2}\right)\left(\frac{S_{d}}{S_{v}}\right)^{2} \\
& P_{i o u t}^{d}=\operatorname{Pr}\left(S I R_{i}^{d} \leq S I R_{t h}^{d}\right) \\
& =\operatorname{Pr}\left(\sum_{j=1}^{N_{v}^{i}} a_{j}^{v} \frac{S_{v}}{S_{d}}+\sum_{j=1}^{N_{d}^{i}-1} a_{j}^{d} m_{d}+\left(m_{d}-1\right)+x \frac{S_{v}}{S_{d}}\right. \\
& \left.+y m_{d}+\frac{I_{v}^{i}+I_{d}^{i}+N_{0}}{S_{d}} \geq \frac{w / R_{b}}{S I R_{t h}^{d}}\right) \\
& =\sum_{k_{d}=0}^{N_{d}^{i}-1} \sum_{k_{v}=0}^{N_{v}^{i}}\left(\begin{array}{c}
N_{d}^{i}-1 \\
k_{d}
\end{array}\right)\left(\begin{array}{c}
N_{v}^{i} \\
k_{v}
\end{array}\right) \alpha_{v}^{k_{v}}\left(1-\alpha_{v}\right)^{N_{v}^{i}-k_{v}} \alpha_{d}^{k_{d}}\left(1-\alpha_{d}\right)^{N_{d}^{i}-1-k_{d}} \\
& Q\left(\frac{\mu_{2}}{\sqrt{\sigma_{3}^{2}+\sigma_{4}^{2}}}\right)
\end{aligned}
$$

where,

$\mu_{2}=\frac{w / R_{b}}{S I R_{t h}^{d}}-\frac{N_{0}}{S_{d}}-k_{v} \frac{S_{v}}{S_{d}}-k_{d} m_{d}-\left(m_{d}-1\right)-\left(\mu_{v} \frac{S_{v}}{S_{d}}+\mu_{d}\right)-\lambda_{v} T_{s} \frac{S_{v}}{S_{d}}-\lambda_{d} T_{s} m_{d}$ 


$$
\begin{gathered}
\sigma_{3}^{2}=\left(\lambda_{v} T_{s}+\sigma_{v}^{2}\right)\left(\frac{S_{v}}{S_{d}}\right)^{2} \\
\sigma_{4}^{2}=\lambda_{d} T_{s} m_{d}^{2}+\sigma_{d}^{2}
\end{gathered}
$$

Similarly, from Equation (6) and (7), we can obtain the outage probability at the adjacent BS $h$ :

$$
\begin{aligned}
P_{h_{\text {out }}^{v}}^{v}= & \operatorname{Pr}\left(S I R_{h}^{v} \leq S I R_{\text {th }}^{v}\right) \\
= & \operatorname{Pr}\left(\sum_{j=1}^{N_{v}^{h}-1} a_{j}^{\prime v}+\sum_{j=1}^{N_{d}^{h}} a_{j}^{\prime} m_{d} \frac{S_{d}}{S_{v}}+\frac{I_{v}^{\prime}+I_{d}^{\prime}+N_{0}}{S_{v}} \geq \frac{w / R_{b}}{S I R_{t h}^{v}}\right) \\
= & \sum_{k_{d}=0}^{N_{d}^{h}} \sum_{k_{v}=0}^{N_{v}^{h}-1}\left(\begin{array}{c}
N_{d}^{h} \\
k_{d}
\end{array}\right)\left(\begin{array}{c}
N_{v}^{h}-1 \\
k_{v}
\end{array}\right) \alpha_{v}^{k_{v}}\left(1-\alpha_{v}\right)^{N_{v}^{h}-1-k_{v}} \alpha_{d}^{k_{d}}\left(1-\alpha_{d}\right)^{N_{d}^{h}-k_{d}} \\
& \cdot Q\left(\frac{\mu_{3}}{\sqrt{\sigma_{v}^{\prime 2}+\sigma_{d}^{\prime 2}\left(\frac{S_{d}}{S_{v}}\right)^{2}}}\right)
\end{aligned}
$$

where,

$$
\begin{aligned}
\mu_{3}=\frac{w / R_{b}}{S I R_{t h}^{v}}-\frac{N_{0}}{S_{v}}-k_{v}-k_{d} m_{d} \frac{S_{d}}{S_{v}}-\left(\mu_{v}^{\prime}+\mu_{d}^{\prime} \frac{S_{d}}{S_{v}}\right) \\
P_{h_{\text {out }}^{d}}^{d}=\sum_{k_{d}=0}^{N_{d}^{h}-1} \sum_{k_{v}=0}^{N_{v}^{h}}\left(\begin{array}{c}
N_{d}^{h}-1 \\
k_{d}
\end{array}\right)\left(\begin{array}{c}
N_{v}^{h} \\
k_{v}
\end{array}\right) \alpha_{v}^{k_{v}}\left(1-\alpha_{v}\right)^{N_{v}^{h}-k_{v}} \alpha_{d}^{k_{d}}\left(1-\alpha_{d}\right)^{N_{d}^{h}-1-k_{d}} \\
\cdot Q\left(\frac{\mu_{4}}{\sqrt{\sigma_{v}^{\prime 2}\left(\frac{S_{v}}{S_{d}}\right)^{2}+\sigma_{d}^{\prime 2}}}\right)
\end{aligned}
$$

where,

$$
\mu_{4}=\frac{w / R_{b}}{S I R_{t h}^{d}}-\frac{N_{0}}{S_{d}}-k_{v} \frac{S_{v}}{S_{d}}-k_{d} m_{d}-\left(m_{d}-1\right)-\left(\mu_{v}^{\prime} \frac{S_{v}}{S_{d}}+\mu_{d}^{\prime}\right)
$$

In the above equations, $Q(\cdot)$ is the error function with $Q(z)=\frac{1}{\sqrt{2 \pi}} \int_{z}^{\infty} e^{-t^{2} / 2} d t$.

The blocking probability can be derived from the admission conditions given by Equation (2) and (3). The blocking probability for a new call equals to the probability that the admission conditions are violated. We define the admission probability at the serving BS $i$ as $P_{s}^{i}$, and $P_{s}^{h}$ for the adjacent $\mathrm{BS} h$. From Equation (2) and (3), we have,

$$
P_{s}^{i}=\operatorname{Pr}\left(B_{n e w}+\sum_{j=1}^{N_{v}^{i}} a_{j}^{v} B_{v}+\sum_{j=1}^{N_{d}^{i}} a_{j}^{d} B_{d}+\frac{I_{v}^{i}}{I_{0}}+\frac{I_{d}^{i}}{I_{0}} \leq 0.9 w\right)
$$




$$
P_{s}^{h}=\operatorname{Pr}\left(\sum_{j=1}^{N_{v}^{i}} a^{\prime}{ }_{j}^{v} B_{v}+\sum_{j=1}^{N_{d}^{i}} a_{j}^{\prime}{ }_{j}^{d} B_{d}+\frac{S_{n e w}^{i}}{I_{0}} \frac{l_{j}(h)}{l_{j}(i)}+\frac{I_{v}^{h}}{I_{0}}+\frac{I_{d}^{h}}{I_{0}} \leq 0.9 w\right)
$$

Then, the blocking probability for a new call in its serving cell $i$ can be expressed as follows:

$$
P_{\text {blocking }}^{i}=1-\left(P_{s}^{i} \prod_{h=1}^{H} P_{s}^{h}\right)
$$

If the new call is the voice service, $B_{\text {new }}$ in the above two admission probability expressions should be $B_{v}=S I R_{t h}^{v} R_{v}$; for a data service call, it is replaced by $B_{d}=S I R_{t h}^{d} R_{d}$.

\section{Numerical Results}

We compare the performance of the CAC method (denoted as Scheme1)which is similar to the proposal in [5] without consideration on the interference brought by the to-be-admitted call to the adjacent cells, with our admission control scheme (denoted as Scheme2). In the following comparison, $8 \mathrm{kbps}$ voice service and $144 \mathrm{kbps}$ (average rate) WWW traffic are considered. The average service time $\left(T_{s}\right)$ for both types of calls is assumed to be 100 seconds. A minimal target SIR of $7 \mathrm{~dB}$ is required by the voice calls to guarantee $10^{-3}$ BER. We denote $R_{b}$ as the basic bandwidth of the sub-channel, $R_{b}=16 \mathrm{kbps}$ is used in our analysis. For the voice traffic with $8 \mathrm{kbps}$ bit rate, one sub-channel is sufficient to support two voice channels. For simplification, Gaussian approximation method is used in both schemes to estimate the interference from the adjacent cells.

Initially, we assume that both voice and data calls in the network have identical BER requirement of $10^{-3}$, which corresponds to the target SIR, and all the neighboring cells have identical traffic loading. In addition, a predefined outage probability of $5 \%$ is given for both voice and data calls. Fig. 1 and Fig. 2 respectively present the simulation results of the capacities in both serving and adjacent cells under different admission control Scheme1 and Scheme2. Comparing to the results of Scheme1 results shown in Fig. 1 Fig. 2 shows that the system using $S$ cheme 2 goes into stable status in both serving and adjacent cells after 200 seconds. It also shows that given a predefined outage probability, more voice and data calls are supported by Scheme1 than Scheme 2 . This is because those to-be-admitted calls which cause more interference to its neighboring cells, are most likely to be rejected in our proposed scheme. Thus, the interference brought by these "bad" calls to the existing connections in both serving and adjacent cells is reduced as much as possible, and the available radio bandwidth is efficiently allocated to support those calls which have lower transmission power under power control and good radio link quality, consequently, the total capacity of the cell cluster using Scheme 2 is larger than that of Scheme1.

Fig. 3 presents the comparison of voice call outage probabilities in both schemes versus the total call arrival rates $\lambda_{v}+\lambda_{d}$, when data calls access the network with different BER requirements of $10^{-2}, 10^{-3}$ and $10^{-6}$. The results 


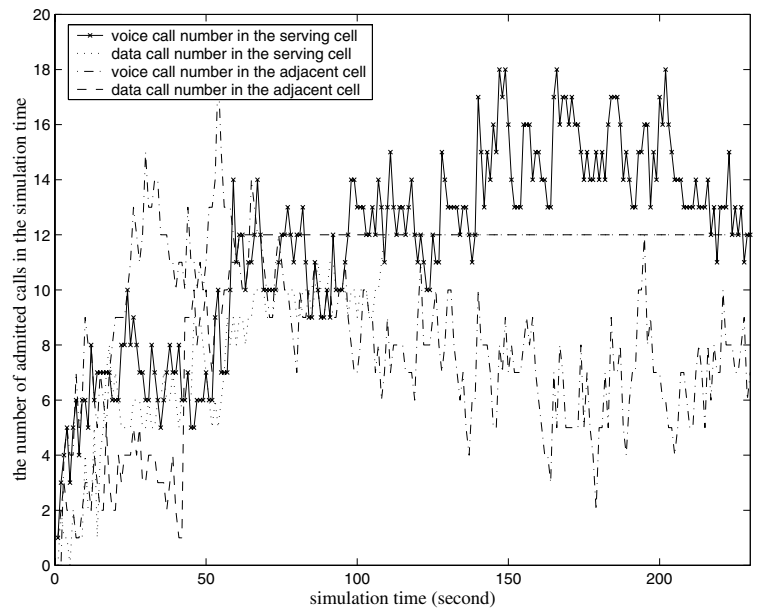

Fig. 1. The system capacity of Scheme 1

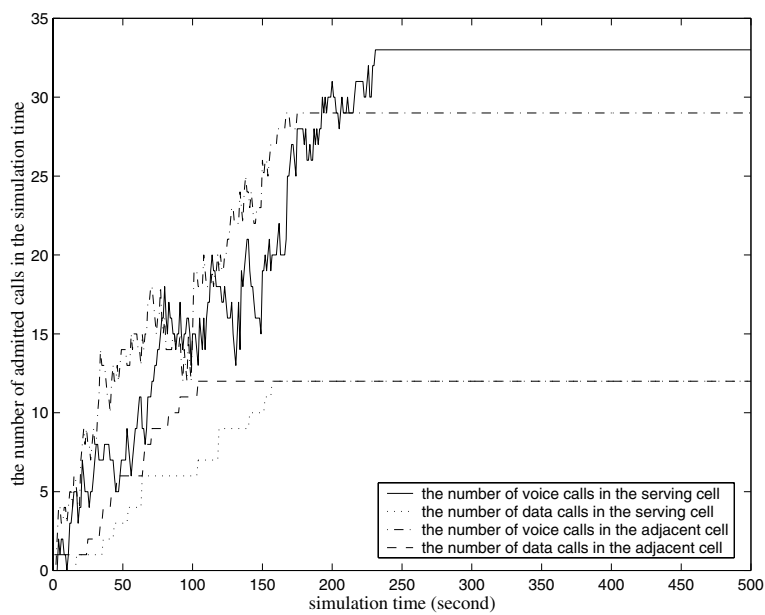

Fig. 2. The system capacity of Scheme 2 .

show that the voice call outage probabilities in Scheme 2 under different data $\mathrm{BER}$ requirements are all lower than those of Scheme1. The reason is that the tobe-admitted calls generated in the adjacent cells are prevented from introducing unacceptable interference to the serving cell, thus the outage probability in our scheme is reduced. Similar performance is also obtained for the data calls. Fig. 3 also shows that high-rate data calls have a significant impact on the QoS of the low-rate voice calls. As the data BER requirement varies from $10^{-2}$ to $10^{-6}$, the voice call outage probability increases. It shows that admitting data calls with higher QoS requirements will degrade the QoS of the other services, because 
more interferences will be introduced to other services from each sub-channel of the data call.

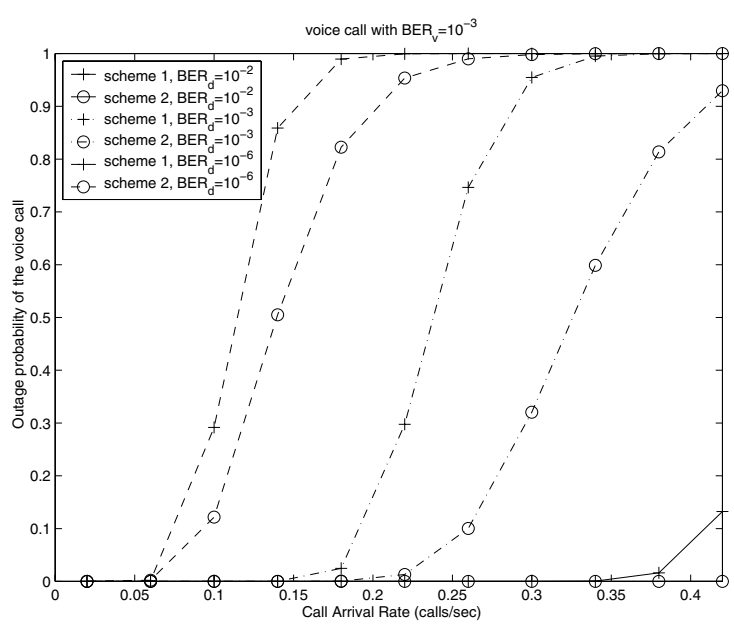

Fig. 3. Comparison of the voice call outage probability in the serving cell.

Fig. 4 presents the call blocking probability in Scheme1 and Scheme2, when both voice and data calls access the network with BER equal to $10^{-3}$. The results in Fig. 4 show that Scheme 2 outperforms the call blocking probabilities in both types of services. In Scheme1, similar call blocking probabilities are expected by both voice and data calls, but the outage probabilities for both types of calls have been increased as in Fig. 3 Scheme 2 gives a higher call blocking probability to the data calls when the network is heavily loaded, while the outage probability of the voice call is lower than that of Scheme1 in Fig. 3. Since the to-be-admitted calls which could introduce unacceptable interference to the neighboring cells are rejected, the total interference measured within the radio link bandwidth is minimized. Accordingly, given the same blocking probability, Scheme 2 can support larger number of high-rate data calls with the guaranteed QoS than Scheme1.

Finally, we consider the case of different traffic loading amongst the cells. We assume that the traffic loading in the serving cell is twice as high as that in the adjacent cells. Under this assumption, the outage probabilities for different offered loads are compared between Scheme1 and Scheme2 in Fig. 5. Fig. 5] shows that the outage probabilities in Scheme1 are higher, but the values of Scheme2 are approaching zero in both hot spot (serving cell) and adjacent cells. This is because $S c h e m e 2$ can prevent the increase in interference from the admitted "bad" calls in the adjacent cells, so as to balance the outage probabilities amongst the cells in a cluster and avoid serious QoS deterioration in the heavily loaded cells. 


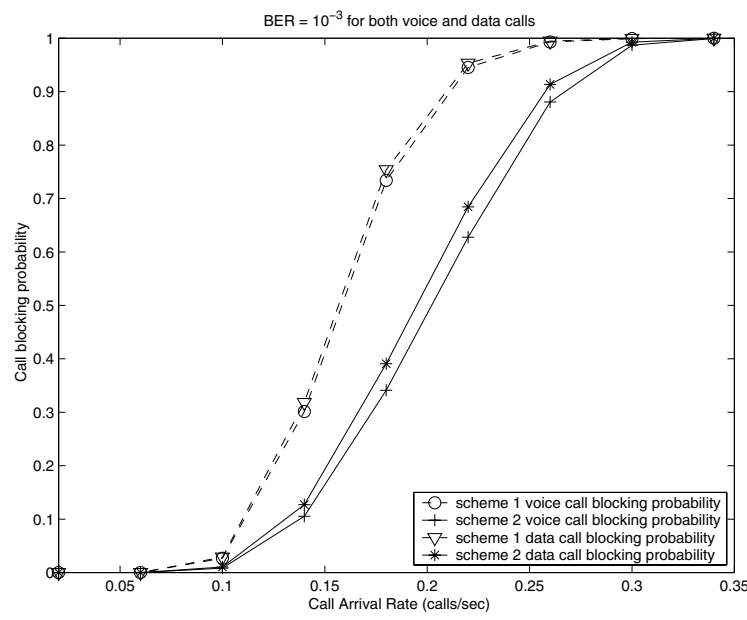

Fig. 4. Comparison of the call blocking probability.

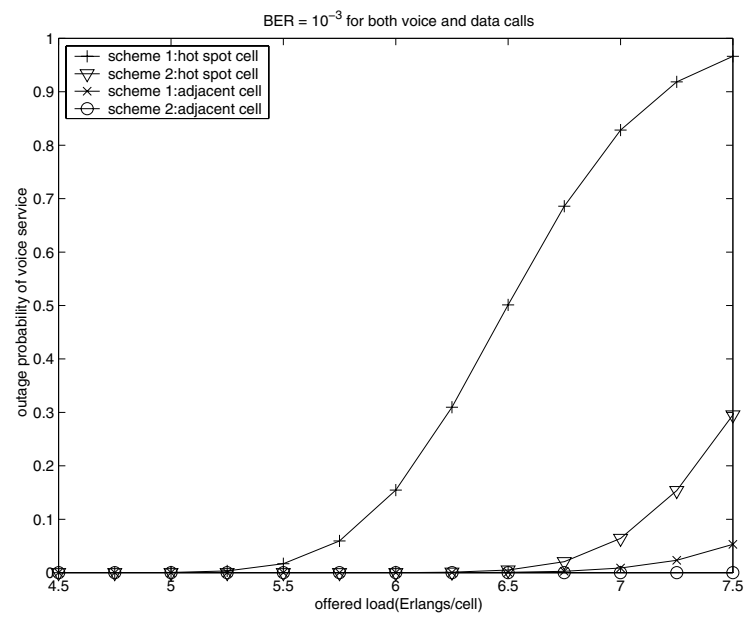

Fig. 5. Outage probability of the voice call under different traffic loading.

\section{Conclusion}

The admission control scheme proposed in this paper for 3G mobile networks is based on the estimated interferences and differentiated QoS requirements for different services. In order to achieve the optimization of QoS over the radio access network, the inter-cell interference and the impacts caused by the to-beadmitted calls to the QoS of the existing connections in the adjacent cells are also considered in our scheme. Under our proposed admission control scheme, the higher admission probability is given to those calls close to their serving BS or experiencing lower path-loss. On the other hand, the calls locating at the edge 
of the serving cell and having large path-loss to the serving BS will introduce exceeded interference to adjacent cells. This kind of calls have a higher blocking probability. Comparisons of the proposed scheme with another scheme which does not take the effects of the to-be-admitted calls into account indicates that the proposed scheme performs better in outage and blocking probabilities.

In our scheme, the issue of call hand-off or terminal mobility is not considered. Further study is under way to take this issue into consideration for admission control in a CDMA based 3G system.

Acknowledgment. The work described in this paper was supported by the City University of Hong Kong under Strategic Research Grant 7001124.

\section{References}

1. Patel G., Dennett S.: The 3GPP and 3GPP2 Movements Toward an All-IP Mobile Network. IEEE Pers. Commun., Vol. 7, Issue 4.8 (2000) 62-64

2. Liu Z., Zarki M. E.: SIR Based Call Admission Control for DS-CDMA Cellular Systems. IEEE J. Select. Areas Commun., Vol. 12, No. 4. 5 (1994) 638-644

3. Kim I. M., Shin B. C., Lee D. J.: SIR-Based Call Admission Control by Intercell Interference Prediction for DS-CDMA Systems. IEEE Communications Letters, Vol. 4, No. 1.12000 29-31

4. Comaniciu C., Mandayam N. B.: QoS Guarantees for Third Generation (3G) CDMA System via Admission and Flow Control. Proceedings, IEEE Veh. Technol. Conf.. (2000) 249-256

5. Shen D., Ji C.: Admission Control of Multimedia Traffic for Third Generation CDMA Network. Proceedings, IEEE INFOCOM 2000. 3 (2000) 1077-1086

6. Gilhousen K. S., Jacobs I. M., Padovani R., Viterbi A. J., Weaver L. A., Wheatley C. E.: On the Capacity of a Cellular CDMA System. IEEE Trans. Veh. Technol., Vol. 40, No. 2. 5 (1991) 303-312

7. Viterbi A. M., Viterbi A. J.: Erlang Capacity of a Power Controlled CDMA System. IEEE J. Select. Areas Commun., Vol. 11, No. 6. 8 (1993) 892-990

8. Ayyagari D., Ephremides A.: Cellular Multicode CDMA Capacity for Integrated (Voice and Data) Services. IEEE J. Select. Areas Commun., Vol. 17, No. 5.5 (1999) 928-938

9. Molina M., Castelli P., Foddis G.: Web Traffic Modeling Exploiting TCP Connections' Temporal Clustering through HTML-REDUCE. IEEE Network. 5/6 (2000) 46-55 